\title{
Characterization of a profilin-like protein from Fasciola hepatica
}

\author{
Jessica Wilkie $^{1,2}$, Timothy C Cameron ${ }^{1,2}$, Travis Beddoe ${ }^{\text {Corresp. 1,2 }}$ \\ ${ }^{1}$ Centre for Livestock Interactions with Pathogens (CLiP), La Trobe University, Bundoora, Victoria, Australia \\ 2 Department of Animal, Plant and Soil Science and Centre for AgriBioscience (AgriBio), La Trobe University, Bundoora, Victoria, Australia \\ Corresponding Author: Travis Beddoe \\ Email address: t.beddoe@latrobe.edu.au
}

Fasciola hepatica is the causative agent of fasciolosis, an important disease of humans and livestock around the world. There is an urgent requirement for novel treatments for $F$. hepatica due to increasing reports of drug resistance appearing around the world. The outer body covering of $F$. hepatica is referred to as the tegument membrane which is of crucial importance for the modulation of the host response and parasite survival; therefore, tegument proteins may represent novel drug or vaccine targets. Previous studies have identified a profilin-like protein in the tegument of $F$. hepatica. Profilin is a regulatory component of the actin cytoskeleton in all eukaryotic cells, and in some protozoan parasites, profilin has been shown to drive a potent IL-12 response. This study characterized the identified profilin form $F$. hepatica (termed FhProfilin) for the first time. Recombinant expression of FhProfilin resulted in a protein approximately 14 kDa in size which was determined to be dimeric like other profilins isolated from a range of eukaryotic organisms. FhProfilin was shown to bind poly-L-proline (pLp) and sequester actin monomers which is characteristic of the profilin family; however, there was no binding of FhProfilin to phosphatidylinositol lipids. Despite FhProfilin being a component of the tegument, it was shown not to generate an immune response in experimentally infected sheep or cattle. 


\section{Characterization of a profilin-like protein from}

\section{Fasciola hepatica.}

3

4

5 Jessica Wilkie ${ }^{1,2}$, Timothy C. Cameron ${ }^{1,2}$, Travis Beddoe ${ }^{1,2}$,

6

$7 \quad{ }^{1}$ Department of Animal, Plant and Soil Science, La Trobe University, Victoria 3086, Australia.

$8{ }^{2}$ Centre for Livestock Interactions with Pathogens (CLiP), La Trobe University, Victoria 3086, 9 Australia.

10

11 Corresponding Author:

12 Travis Beddoe

135 Ring Rd, Bundoora, Victoria, 3086, Australia

14 Email address: t.bedddoe@latrobe.edu.au 


\section{Abstract}

17 Fasciola hepatica is the causative agent of fasciolosis, an important disease of humans and

18 livestock around the world. There is an urgent requirement for novel treatments for $F$. hepatica

19 due to increasing reports of drug resistance appearing around the world. The outer body covering

20 of $F$. hepatica is referred to as the tegument membrane which is of crucial importance for the

21 modulation of the host response and parasite survival; therefore, tegument proteins may

22 represent novel drug or vaccine targets. Previous studies have identified a profilin-like protein in

23 the tegument of $F$. hepatica. Profilin is a regulatory component of the actin cytoskeleton in all

24 eukaryotic cells, and in some protozoan parasites, profilin has been shown to drive a potent IL-

2512 response. This study characterized the identified profilin form F. hepatica (termed FhProfilin)

26 for the first time. Recombinant expression of $F h$ Profilin resulted in a protein approximately 14

$27 \mathrm{kDa}$ in size which was determined to be dimeric like other profilins isolated from a range of

28 eukaryotic organisms. FhProfilin was shown to bind poly-L-proline ( $\mathrm{pLp}$ ) and sequester actin

29 monomers which is characteristic of the profilin family; however, there was no binding of

30 FhProfilin to phosphatidylinositol lipids. Despite FhProfilin being a component of the tegument,

31 it was shown not to generate an immune response in experimentally infected sheep or cattle. 


\section{Introduction}

34 Fasciolosis is a worldwide distributed zoonotic infectious disease and constitutes a serious 35 worldwide problem in both humans and livestock (Mas-Coma et al. 2018; Mas-Coma et al.

36 2019). There are two major pathogens of fasciolosis; Fasciola hepatica and F. gigantica, which are commonly referred to as liver fluke. The control of fasciolosis has relied upon chemotherapy, predominanantly with the drug triclabendazole. However, due to an over-reliance on this drug in recent years, resistance to triclabendazole has developed (Fairweather 2009; Kelley et al. 2016). As triclabendazole is the only drug that will kill both the juvenile and adult life stages of liver flukes, there is an urgent need for the development of novel treatments. For this reason, vaccine development is seen as a sustainable method for the control of Fasciola spp. (Molina-Hernandez et al. 2015; Toet et al. 2014).

The current $F$. hepatica vaccine candidates being investigated have shown only moderate protection against $F$. hepatica infection (Toet et al. 2014). The development of an effective vaccine will require a thorough understanding of the host-parasite interactions (Cwiklinski \& Dalton 2018; Cwiklinski et al. 2018). While the excretory-secretory (ES) products of $F$. hepatica have been thoroughly investigated as vaccine antigens with little sustained success, the tegument surface represents the key interface in host-parasite interactions, performing numerous functions for the parasite such as nutrient absorption, sensory input and protection from the host immune response (Halton 2004). Host antibodies have been demonstrated to have an ability to bind to the tegument antigens of $F$. hepatica (Howell and Sandeman 1979; Hanna 1980; Sulaiman et al. 2016; Cameron et al. 2017), suggesting that tegument-directed vaccine candidates warrant further investigation. Despite this, there are few reported cases of tegument-directed vaccines. Tegument proteins as vaccine targets in other helminth parasites such as Schistosoma mansoni show immense promise, with phase 1 clinical trials in progress (Fonseca et al. 2015; Merrifield et al. 2016; Molehin 2020) and we propose that tegument proteins represent potential novel vaccine antigens for F. hepatica control (Hanna et al. 1988; Sobhon et al. 1998).

The tegument surface of Fasciola spp. is a dynamic syncytial layer surrounded by a glycocalyx (Hanna 1980; Lammas \& Duffus 1983). Various groups in recent times have attempted to characterise the proteome (Hacariz et al. 2012; Ravida et al. 2016; Wilson et al. 2011) and 
64 immunoproteome (Cameron et al. 2017) of $F$. hepatica using mass spectrometry. The proteome 65 of an enriched tegument extract of $F$. hepatica revealed a range of proteins shared with the

66

67

68

69

70

71

72

73

74

75

76

77

78

79

80

81

82

83

84

85

86

87

88

89

90 91

\section{Materials \& Methods}

93 Cloning and phylogenetic analysis of Fasciola hepatica profilin (FhProfilin)

schistosome tegument including annexins, tetraspanins, carbonic anhydrase and an orthologue of a host protein (CD59) (Wilson et al. 2011). A second study enriched tegument glycoproteins using immobilised lectin chromatography to identify over 369 glycoproteins with a broad range of functions such as proteases, protease inhibitors, paramyosin, venom allergen-like protein II and enolase (Ravida et al. 2016). There is an over-abundance of vaccine antigen candidates from the tegument and to narrow down potential candidates, a recent study used a novel ex vivo immunoproteomic technique whereby contact with purified host IgG from infected animals, the flukes will slough (i.e. sheds) its tegument proteins after antibody binding has occurred (Cameron et al. 2017). This immunosloughate identified 38 proteins that could be potential vaccine antigens, (Cameron et al. 2017). Unsurprisingly, all these tegument proteomic studies identified a large number of cytoskeletal elements such as tubulin, actin and profilin that could be potential vaccine/drug targets due to the crucial function of the Fasciola tegument.

Profilins are small actin-binding proteins that are involved in the regulation of actin polymerization by sequestering actin and ADP/ATP exchange (Krishnan \& Moens 2009; PintoCosta \& Sousa 2020). In addition, they are involved in cell signalling between the cell membrane and cytoskeleton by interacting with polyphosphoinositides (PPI) and proline-rich domain containing proteins (Krishnan \& Moens 2009; Pinto-Costa \& Sousa 2020). In particular, profilin from apicomplexan protozoan parasites such as Toxoplasma gondii have been shown to generate a potent IL-12 response in murine DCs activated through TLR11; as such, profilin from various apicomplexan protozoan parasites have been trialled as vaccine antigens (D'Angelo et al. 2009; Mansilla \& Capozzo 2017; Tang et al. 2018; Yarovinsky et al. 2005). Here we describe the identification and biochemical characterization via bioinformatics, phospholipid binding, actin polymerisation and poly-L-proline affinity that a tegument protein from $F$. hepatica can be classified as belong to the profilin family and we describe its potential use as vaccine candidate. 
94 The native $F$. hepatica profilin sequence (accession number D915_008168) was chemically 95 synthesized and cloned (Bioneer, USA) via NdeI and XbaI sites into a modified pET-28 vector, 96 resulting in an open reading frame containing an N-terminal hexahistidine tag followed by an 97 HRV 3C protease cleavage site and the FhProfilin sequence. The Phylogeny.fr program was used 98 to compare FhProfilin with identified profilins from other parasitic species and construct a 99 phylogenetic tree based on multiple alignments and a neighbour-joining method as well as to 100 estimate the confidence value of the branching patterns (Dereeper et al. 2008).

101

102

Recombinant FhProfilin expression

103

Plasmid DNA containing the FhProfilin sequence was transformed into BL21 (DE3) E. coli cells

104

105

106

107

108

109

110

111

112

113

114

115

116

117

118

119

120

121

122

123

124 and plated onto a Luria-Bertani (LB) agar plate containing kanamycin $\left(50 \mu \mathrm{g} \mathrm{ml}^{-1}\right)$. A single colony was inoculated into a starter culture and grown overnight in $10 \mathrm{ml} \mathrm{LB}$ medium containing $50 \mu \mathrm{g} \mathrm{ml}^{-1}$ kanamycin with shaking at $225 \mathrm{rpm}$. The starter culture was used at a 1:100 dilution to inoculate $400 \mathrm{ml}$ of fresh LB medium containing $50 \mu \mathrm{g} \mathrm{ml} \mathrm{m}^{-1}$ kanamycin and grown at $37^{\circ} \mathrm{C}$ until the optical density at wavelength $600 \mathrm{~nm}(\mathrm{OD} 600)$ reached 0.5 . Expression of FhProfilin was induced with $0.5 \mathrm{mM}$ IPTG (isopropyl $\beta$-D-1-thiogalactopyranoside) and the cells were allowed to grow for a further $4 \mathrm{~h}$ at $37^{\circ} \mathrm{C}$. The cells were collected by centrifugation at $6000 \mathrm{~g}$ for 10 min at $4{ }^{\circ} \mathrm{C}$ and were stored at $-20^{\circ} \mathrm{C}$.

\section{Recombinant FhProfilin purification}

The frozen cell pellet was thawed on ice and resuspended in $2 \mathrm{ml}$ of lysis buffer $(50 \mathrm{mM}$ $\mathrm{NaH}_{2} \mathrm{PO}_{4}, 300 \mathrm{mM} \mathrm{NaCl}$ and $10 \mathrm{mM}$ imidazole at $\mathrm{pH}$ 8.0) per gram of wet cell weight. After thawing, $200 \mu \mathrm{L}$ of $25 \mathrm{mg} / \mathrm{mL}$ lysozyme and $200 \mu \mathrm{L}$ of $2 \mathrm{mg} / \mathrm{ml}$ DNase was added and incubated on ice for 30 minutes. The solution was sonicated with $3 \mathrm{~mm}$ microprobe using a Sonics Vibracell VCX 130PB at 25-30\% amplitude with $30 \mathrm{sec}$ bursts on ice for a total sonication time of three minutes, with 30 seconds of rest in between each burst. The cell debris was removed by centrifugation at $30,000 \mathrm{~g}$ for $20 \mathrm{~min}$ at $4^{\circ} \mathrm{C}$. The supernatant was added to 1 $\mathrm{mL}$ of $50 \%(\mathrm{w} / \mathrm{v}) \mathrm{Ni}$-Sepharose resin (Clontech) pre-washed with 5 column volumes of lysis buffer and incubated for $1 \mathrm{~h}$ with gentle shaking at $4^{\circ} \mathrm{C}$. The lysate-nickel Sepharose mixture was loaded into a gravity flow column and the flow through collected. The column was washed with 2 column volumes of wash buffer $\left(50 \mathrm{mM} \mathrm{NaH}_{2} \mathrm{PO}_{4}, 300 \mathrm{mM} \mathrm{NaCl}\right.$ and $20 \mathrm{mM}$ imidazole, 
$125 \mathrm{pH} \mathrm{8.0)}$ and eluted with $8 \mathrm{ml}$ of elution buffer $\left(50 \mathrm{mM} \mathrm{NaH}_{2} \mathrm{PO}_{4}, 300 \mathrm{mM} \mathrm{NaCl}\right.$ and $250 \mathrm{mM}$

126 Imidazole, $\mathrm{pH}$ 8.0) and collected as $2 \mathrm{ml}$ fractions. Each step of the purification process was

127 validated by visualization of protein fractions using SDS-PAGE.

128

129 Fractions containing FhProfilin were pooled and concentrated to $5 \mathrm{ml}$ using Amicon

130 ultracentrifugal filters (3 kDa molecular-weight cutoff; Millipore). The partially purified

131 FhProfilin was further purified by size-exclusion chromatography with a Superdex S75 16/60

132 gel-filtration column (GE Healthcare Life Sciences) equilibrated in TBS (10 mM Tris-HCL and

$133300 \mathrm{mM} \mathrm{NaCl}, \mathrm{pH}$ 8.0) using an AKTA Basic fast protein liquid-chromatography (FPLC)

134 system at $1 \mathrm{ml} / \mathrm{min}$. The molecular weight, purity and identity of the FhProfilin preparation were

135 confirmed by SDS-PAGE and Western blotting.

136

\section{Actin affinity assay}

138 The ability of $F h$ Profilin to bind and sequester actin was investigated. Actin $(5 \mu \mathrm{M})$ derived from 139 bovine muscle (Sigma, USA) was induced to polymerise with $1 \mathrm{mM} \mathrm{MgCl} 2$ and $0.15 \mathrm{M} \mathrm{KCl}$. FhProfilin was added in molar ratios of 1:1, 1:2 and 1:4 molar ratios to the polymerized actin in total volume of $150 \mu \mathrm{l}$ and incubated at room temperature for 2-3 $\mathrm{h}$. After incubation, the actinprofilin mixtures were centrifuged at $100,000 \mathrm{x} g$ for $30 \mathrm{~min}$ at $20^{\circ} \mathrm{C}$. Equal amounts of the supernatants and pellets fractions were analysed by SDS-PAGE.

145 Phospholipid affinity assay

146 PIP Strips ${ }^{\mathrm{TM}}$ (Echelon Biosciences Incorporated, USA) were used to assess the specificity of

147 FhProfilin for associating with various phospholipids. Each membrane has been spotted with 15 148 assorted phospholipids at 100 pmol in each spot. The membrane was blocked with $5 \mathrm{~mL}$ of PBS 149 (50 mM Sodium phosphate, $150 \mathrm{mM} \mathrm{NaCl}, \mathrm{pH}$ 7.4) plus $3 \%$ (w/v) skim milk blocking solution 150 and gently shaken for $1 \mathrm{~h}$ at room temperature. The blocking solution was then discarded and 151 various concentrations of FhProfilin, starting with an initial concentration of $20 \mu \mathrm{g} / \mathrm{mL}$ up to 500 $152 \mu \mathrm{g} / \mathrm{mL}$, was added to $10 \mathrm{~mL}$ of PBS plus $3 \%(\mathrm{w} / \mathrm{v})$ skim milk and gently shaken at room 153 temperature for $1 \mathrm{~h}$. 
155 For the positive control, $5 \mu \mathrm{g} / \mathrm{mL}$ of $\mathrm{PI}(4,5) \mathrm{P}_{2}$ Grip $^{\mathrm{TM}}$ protein in $5 \mathrm{~mL}$ of PBS-T (50 mM Sodium 156 phosphate, $150 \mathrm{mM} \mathrm{NaCl}, 0.05 \%$ (v/v) Tween $20 \mathrm{pH} 7.4)$ plus $3 \%(\mathrm{w} / \mathrm{v}) \mathrm{BSA}$ was used. The 157 protein solution was discarded, and the membrane was washed three times with $5 \mathrm{~mL}$ of PBS 158 with $5 \mathrm{~min}$ of gentle shaking for each wash. After washing the test strip, anti-His HRP159 conjugated antibody (R\&D systems, USA) was diluted to 1: 10,000 in PBS with 3\% (w/v) skim 160 milk for FhProfilin and added to the membrane and incubated for $1 \mathrm{~h}$ at room temperature with

161 162 163

164 165 166

167

168 169

170

171

172

173

174

175

176

177

178

179

180

181

182

183

184 gentle shaking. For the positive control strip, anti-GST-HRP antibody (GenScript, USA) was diluted to 1: 2,000 in PBS-T 3\% (w/v) BSA. The antibody solution was discarded, and the membrane washed as previously stated. For both $F h$ Profilin and positive control samples, detection was performed incubating the mebrane with $5 \mathrm{ml}$ of Clarity ECL substrate (Bio-Rad Laboratories, USA) for $5 \mathrm{~min}$ and imaged using the C-DiGiT blot scanner (Li-Cor).

\section{Poly-L-proline affinity assay}

The affinity of $F h$ Profilin for proline-rich domains was investigated. Poly-L-proline (pLp) sepharose was prepared by coupling $50 \mathrm{mg}$ of pLp (Sigma-Aldrich) to $1 \mathrm{~g}$ of cyanogen bromideactivated sepharose resin (Sigma-Aldrich). The pLp was dissolved in $4 \mathrm{~mL}$ of ice cold deionized water and the sepharose was resuspended in $8 \mathrm{~mL}$ of $250 \mathrm{mM}$ sodium carbonate to make a $50 \%$ slurry. The pLp was added to the 50\% slurry and stirred for $2 \mathrm{~h}$ at room temperature. The mixture was transferred to a cold room and stirred at $4^{\circ} \mathrm{C}$ overnight. The reaction was quenched with $1.2 \mathrm{~mL}$ of $10 \mathrm{X}$ bead buffer $(1 \mathrm{M} \mathrm{NaCl}, 1 \mathrm{M}$ glycine and $100 \mathrm{mM}$ Tris). The resin was washed in a Buchner funnel with $500 \mathrm{~mL}$ of deionized water, dried and stored at $4^{\circ} \mathrm{C}$ in $1 \mathrm{X}$ storage buffer (10 mM Tris at $\mathrm{pH} 7.5,50 \mathrm{mM} \mathrm{KCl,} 1 \mathrm{mM}$ EDTA and 0.002\% (w/v) sodium azide). $50 \mu \mathrm{L}$ aliquots of the $50 \%$ pLp sepharose slurry with PBS was incubated with $10 \mathrm{nM}$ of purified FhProfilin for test samples and $10 \mathrm{nM}$ of BSA for control samples. Each sample was incubated at room temperature for $15 \mathrm{~min}$ and analysed by SDS PAGE. To quantify the binding effect of FhProfilin with poly-L-proline (pLp), different concentrations of FhProfilin, from 1-10 $\mu \mathrm{g} / \mu \mathrm{L}$, were incubated with the poly-L-proline sepharose beads for 15 minutes and analyzed by SDS-PAGE.

\section{Immune profile of $F$ Profilin in sheep and cattle sera}


185

186

187

188

189

190

191

192

193

194

195

196

197

198

199

200

201

202

203

204

205

206

207

208

209

210

211

212

213

214

215

216

217

218

219

216

$F$. hepatica were obtained from the abattoir and whole worm extract (WE) was prepared as previously reported (Swan et al. 2019). Native GST was purified as previously described by (Wijffels et al. 1992). $40 \mu \mathrm{g}$ of WE, purified FhProfilin (3 $\mu \mathrm{g})$ and native GST (3 $\mu \mathrm{g})$ were loaded onto an SDS-PAGE and transferred to PVDF membrane (Bio-Rad Laboratories, USA) using a Trans-Blot ${ }^{\circledR}$ Turbo ${ }^{\mathrm{TM}}$ Transfer System (Bio-Rad Laboratories, USA). After blocking with $5 \%(\mathrm{w} / \mathrm{v})$ skim milk, pooled sera from experimentally infected Merino sheep (12 animals pooled, 6 weeks post-infection, infected with 200 metacercariae), Indonesian Thin Tailed (ITT) sheep (15 animals pooled, 6 weeks post-infection, infected with 200 metacercariae and cattle (6 animals pooled from 125 days post-infection, infected with 350 metacercariae (kindly donated by Prof. Terry Spithill) were incubated at a 1: 4,000 dilution with the blots in 5\% (w/v) skim milk with PBS-T for $1 \mathrm{~h}$. Membranes were washed three times with PBS-T and were then incubated with either anti-sheep HRP-conjugated IgG or anti-bovine HRP-conjugated IgG (Sigma-Aldrich) diluted to 1: 4,000 in 5\% (w/v) skim milk in PBS-T for $1 \mathrm{~h}$. Membranes were washed again as above and thens were visualized by Clarity ECL substrate (Bio-Rad Laboratories, USA) and C-DiGiT blot scanner (Li-Cor) according to the manufacturer's instructions.

\section{Results and Discussion}

\section{Bioinformatic analysis of the Fasciola hepatica profilin gene}

The profilin gene identified (accession number D915_008168; GEVX010111281) in F. hepatica (FhProfilin) has an open reading frame (ORF) of $393 \mathrm{bp}$, encoding a 130 amino acid protein with a predicted molecular mass of $14 \mathrm{kDa}$ and a predicted isoelectric point of 5.35, which was predicted using the "ProtParam" tool from ExPASy Bioinformatics Resource Portal (https://web.expasy.org/protparam/). A domain search using InterPro

(http://www.ebi.ac.uk/interpro/), revealed the presence of a conserved profilin domain, containing putative actin binding sites (Fig. 1a). Structural homology modeling revealed that FhProfilin is structural indentical to Saccharomyces cerevisiae profilin with conservation of residues involved in proline binding however residues in a putative phosphatidylinositol 4,5bisphosphate (PIP2)-interaction site are not conserved (Fig. 1b; Fig S1).

In a phylogenetic analysis of profilin proteins from other parasite species, it was revealed that FhProfilin clustered in a clade alongside other trematode species with identities ranging from $31.1 \%$ (Schistosoma japonicum) to $41.5 \%$ (Clonorchis sinensis), while profilins from apicomplexan parasites clustered in a separate clade (Fig. 2). 


\section{Protein expression and purification of $\boldsymbol{F h P r o f i l i n}$}

222 FhProfilin was recombinantly expressed in E. coli and purified via immobilized metal-ion

223 affinity chromatography, and when visualized using SDS-PAGE resulted in a single protein with

224 an expected molecular mass of $14 \mathrm{kDa}$ showing that $F h$ Profilin was successfully expressed and 225 purified as a soluble protein (Fig. 2a). To further purify and characterize FhProfilin it was 226 subjected to size-exclusion chromatography. A typical trace (Fig. 2b) revealed that FhProfilin 227 elutes at the volume of approximately $75 \mathrm{ml}$ which corresponds to a molecular weight of 228 approximately $30 \mathrm{kDa}$, suggesting that FhProfilin is a dimer in solution. Several previous studies 229 have reported that profilin from different species such as yeast, birch pollen and humans can 230 form dimers and tetramers in solution (Babich et al. 1996; Mittermann et al. 1998; Wopfner et al. 231 2002). The oligomeric state of profilin is important for the function of certain profilins, for 232 example, the allergenic potential of birch pollen is higher if profilin is in a dimeric state (Mares233 Mejia et al. 2016). In addition, interactions with phosphoinositides and poly-L-proline (pLp) is 234 regulated by an oligomeric form of profilin, with dimers having a weaker affinity to $\mathrm{pLp}$ than 235 tetrameric profilin (Korupolu et al. 2009), suggesting the oligomeric state of FhProfilin may 236 regulate its function.

237

\section{Biochemical characterization of FhProfilin}

239 Profilins are characterized by having three major biochemical functions (Krishnan \& Moens 240 2009; Moreau et al. 2017; Moreau et al. 2020); firstly, profilins bind to and sequester actin 241 monomers, therefore affecting how actin filaments polymerize. Secondly, profilins have an 242 affinity for proline-rich domains contained within their interacting ligands and lastly, they have 243 an affinity for phosphatidylinositol lipids that dissociate the actin:profilin complexes.

245 To determine if FhProfilin is able to perform the classic functional profile of other profilins, 246 several in vitro assays were performed. Recombinant FhProfilin was added to polymerizing 247 bovine actin at different ratios and monomeric actin was separated from polymerized actin by 248 centrifugation. At all ratios of actin:profilin, the majority of actin appeared in the soluble 249 supernatant fraction with very little polymerized actin being observed in the pellet fractions (Fig. 250 3). This indicates that FhProfilin has a strong ability to sequester actin similar to other profilins 
251 from yeast and humans (Eads et al. 1998; Pinto-Costa \& Sousa 2020). A major defense

252 mechanism of $F$. hepatica is the ability to shed its tegument proteins after binding by antibodies,

253 which is highly dependent on cytoskeletal rearrangement, thus making FhProfilin an ideal

254 vaccine candidate or drug target (Hanna 1980). Human and Plasmodium profilin have been

255 explored as possible drug targets and many of the major drugs against Fasciola target tubulin,

256 thus cytoskeletal components present ideal drug targets (Fairweather et al. 2020; Kumpula \&

257 Kursula 2015; Moens \& Coumans 2015). In the future, the actin-profilin interaction could be

258 explored as possible drug target in Fasciola.

259

260 Profilin interacts with its many ligands via proline-rich sequences (Bjorkegren et al. 1993;

261 Kursula et al. 2008b; Mahoney et al. 1997). The majority of residues involved in polyproline

262 binding are conserved in FhProfilin suggesting it interacts with a variety of partner proteins (Fig.

263 1a). To assess the ability of $F h$ Profilin to interact with polyproline, it was subjected to pulldown

264 assays using polyproline-sepharose beads (Fig. 4). FhProfilin was successfully detected in the

265 bound fraction of the assay, whereas BSA was not (Fig. 4), showing that the interaction with

266 polyproline-sepharose is specific to FhProfilin. Not all FhProfilin was bound to the resin,

267 suggesting either the interaction was weak, or capacity of the resin was exceeded (Fig. 4). It

268 appears that $F$ Profilin may bind to similar physiological substrates as human profilin and

269 therefore is likely to be involved in other cellular functions such as ribonucleoparticle processing

270 (Giesemann et al. 1999), mRNA splicing (Skare et al. 2003) and nuclear export (Stuven et al.

271 2003).

272

273 The ability of profilins to bind phosphatidylinositol lipids is important as it can regulate

274 phosphoinositide metabolism and its ability to move from the membrane to the cytosol where it

275 can interact with actin or other ligands (Chaudhary et al. 1998; Lambrechts et al. 2002). The

276 phosphatidylinositol lipid specificity of $F h$ Profilin was assessed using a mini

277 phosphatidylinositol lipid array (Fig. 5). There was no detectable phosphatidylinositol lipid

278 binding by FhProfilin even at the highest concentration of $500 \mu \mathrm{g} / \mathrm{ml}$ (Fig. 5b). However, the

279 positive control protein Grip supplied with the array was positively identified binding to the

280 appropriate lipid phosphatidylinositol 4, 5-bisphosphate (Fig. 5c) confirming the validity of the

281 assay and suggesting that $F h$ Profilin has very weak or no association with phosphatidylinositol 
282 lipids. It is not surprising that FhProfilin does not bind phosphatidylinositol lipids as only two 283 out of five PIP-2 binding sites are conserved (Fig. S1), which are normally seen in other 284 members of the profilin family (Munkhjargal et al. 2016). Human profilin has been shown to 285 bind to $\mathrm{PI}(3,4) \mathrm{P}_{2}, \mathrm{PI}(3,4) \mathrm{P}_{2}$ and $\mathrm{PI}(3,4,5) \mathrm{P}_{3}$ which is silimar to bovine profilin that binds $286 \mathrm{PI}(3,4,5) \mathrm{P}_{3}$ and $\mathrm{PI}(4,5) \mathrm{P}_{2}$ with some binding to $\mathrm{PI}(3) \mathrm{P}, \mathrm{PI}(4) \mathrm{P}$, and $\mathrm{PI}(5) \mathrm{P}$ (Kursula et al. 2008a; 287 Lu et al. 1996). This different which has been observed between the more closely related 288 profilins from the apicomplexan parasites Plasmodium and T. gondii that Plasmodium profilin 289 can bind phosphatidylinositol lipids (PI(4)P, and PI(5)P) whereas T. gondii profilin cannot 290 (Kucera et al. 2010; Kursula et al. 2008a). The lack of binding of FhProfilin to 291 phosphatidylinositol lipids may suggest the diffenence in phosphatidylinositol lipid metabolism 292 in F. hepatica.

293

294 Immune profile in cattle and sheep

295 Apicomplexan parasitic profilins have the ability to stimulate the immune system via toll-like 296 receptor 11 (TLR11) due to the presence of a parasite-specific surface motif consisting of an 297 acidic loop followed by a long $\beta$-hairpin insert (Fig. S1) (Kucera et al. 2010). Due to this 298 immune modulation activity, profilins have been used as potential vaccine candidates and 299 adjuvants, in particular against T. gondii and Eimeria spp. (Jang et al. 2011a; Jang et al. 2011b; 300 Jang et al. 2011c; Tanaka et al. 2014). To ascertain if FhProfilin was exposed to the host immune 301 system, pooled sera from experimentally Fasciola infected animals was tested via Western Blot 302 (Fig. 6). Immune sera from infected animals consisting of the susceptible sheep breed (Merino), 303 the $F$. gigantica-resistant Indonesian Thin Tail (ITT) sheep and cattle did not recognize the 304 recombinant FhProfilin while native $F$. hepatica glutathione S-transferase (nFhGST), a major parasite excretory-secretory antigen, was recognized (LaCourse et al. 2012). This suggests that Fasciola profilin is not exposed to immune system during infection (Fig. 6). The lack of FhProfilin-specific antibodies from exposed animals is unexpected as profilin from other parasites such Babesia spp. (Munkhjargal et al. 2016) and Schistosoma japonicum do elicit an immune response post infection (Zhang et al. 2008). Further the use of electron microscopy to perform ultrastructural studies to determine where within the tegument FhProfilin is located and whether this protein is exposed to the host immune system would help to assess the validity of $F h$ Profilin as a vaccine candidate. A lack of direct exposure on the tegument should not exclude 
313 FhProfilin as a vaccine candidate, as the "hidden" antigen vaccines are against the nematode

314 Haemonchus contortus (LeJambre et al. 2008; Munn 1997) and the tick Rhiphicephalus

315 microplus (Willadsen \& Kemp 1988) demonstrate the commercial viability of targeting antigens

316 of this nature, as long they are essential in function to the parasite. Profilin is essential for the

317 survival of $P$. falciparum (Kursula et al. 2008a) and necessary for virulence of $T$. gondii (Plattner

318 et al. 2008), suggesting that profilin could also be essential for the survival and pathogenesis of

319 F. hepatica.

320

321 Conclusions

322 Fasciola is a zoonotic infection of worldwide concern which until recently was successfully

323 controlled through the use of triclabendazole; however, the appearance of drug resistant parasites

324 has required the need for the development of a vaccine or new drug targets. We have

325 characterized a putative open reading frame that has homology to the profilin family. Profilin

326 plays an essential role in regulating the actin cytoskeleton in all eukaryotic cells. The

327 recombinant $F h$ Profilin displayed hallmark biochemical features of other profilins by binding to

328 actin and polyproline, however the lack of binding to phosphatidylinositol lipids suggests that

329 phosphatidylinositol lipid metabolism may be different in Fasciola compared to other parasite

330 species. Despite recombinant $F h$ Profilin not being recognized by immune sera from infected

331 animals, the use of $F h$ Profilin as a potential vaccine candidate is worth further investigation due

332 to the predicted critical function of this protein to the parasite's pathogenesis and survivability.

333

334

335

336

337

338

339

340

341

342

343

344

\section{Acknowledgements}

We thank Jaclyn Swan for help with analyzing the Fasciola hepatica genome database, Gemma Zerna for the native GST protein and Prof. Terry Spithill for the donation of sheep and cattle immune sera.

\section{References}

Babich M, Foti LR, Sykaluk LL, and Clark CR. 1996. Profilin forms tetramers that bind to Gactin. Biochem Biophys Res Commun 218:125-131. 10.1006/bbrc.1996.0022 
345

346

347

348

349

350

351

352

353

354

355

356

357

358

359

360

361

362

363

364

365

366

367

368

369

370

371

372

373

374

375

376

377

378

379

380

381

382

383

384

385

386

387

388

389

390

391

392

393

394
Bjorkegren C, Rozycki M, Schutt CE, Lindberg U, and Karlsson R. 1993. Mutagenesis of human profilin locates its poly(L-proline)-binding site to a hydrophobic patch of aromatic amino acids. FEBS Lett 333:123-126. 10.1016/0014-5793(93)80388-b

Cameron TC, Cooke I, Faou P, Toet H, Piedrafita D, Young N, Rathinasamy V, Beddoe T, Anderson G, Dempster R, and Spithill TW. 2017. A novel ex vivo immunoproteomic approach characterising Fasciola hepatica tegumental antigens identified using immune antibody from resistant sheep. Int J Parasitol 47:555-567. 10.1016/j.ijpara.2017.02.004

Chaudhary A, Chen J, Gu QM, Witke W, Kwiatkowski DJ, and Prestwich GD. 1998. Probing the phosphoinositide 4,5-bisphosphate binding site of human profilin I. Chem Biol 5:273-281. 10.1016/s1074-5521(98)90620-2

Cwiklinski K, and Dalton JP. 2018. Advances in Fasciola hepatica research using 'omics' technologies. Int J Parasitol 48:321-331. 10.1016/j.ijpara.2017.12.001

Cwiklinski K, Jewhurst H, McVeigh P, Barbour T, Maule AG, Tort J, O'Neill SM, Robinson MW, Donnelly S, and Dalton JP. 2018. Infection by the Helminth Parasite Fasciola hepatica Requires Rapid Regulation of Metabolic, Virulence, and Invasive Factors to Adjust to Its Mammalian Host. Mol Cell Proteomics 17:792-809. 10.1074/mcp.RA117.000445

D'Angelo JG, Bordon C, Posner GH, Yolken R, and Jones-Brando L. 2009. Artemisinin derivatives inhibit Toxoplasma gondii in vitro at multiple steps in the lytic cycle. $J$ Antimicrob Chemother 63:146-150. 10.1093/jac/dkn451

Dereeper A, Guignon V, Blanc G, Audic S, Buffet S, Chevenet F, Dufayard JF, Guindon S, Lefort V, Lescot M, Claverie JM, and Gascuel O. 2008. Phylogeny.fr: robust phylogenetic analysis for the non-specialist. Nucleic Acids Res 36:W465-469. 10.1093/nar/gkn180

Eads JC, Mahoney NM, Vorobiev S, Bresnick AR, Wen KK, Rubenstein PA, Haarer BK, and Almo SC. 1998. Structure determination and characterization of Saccharomyces cerevisiae profilin. Biochemistry 37:11171-11181. 10.1021/bi9720033

Fairweather I. 2009. Triclabendazole progress report, 2005-2009: an advancement of learning? J Helminthol 83:139-150. 10.1017/S0022149X09321173

Fairweather I, Brennan GP, Hanna REB, Robinson MW, and Skuce PJ. 2020. Drug resistance in liver flukes. Int J Parasitol Drugs Drug Resist 12:39-59. 10.1016/j.jpddr.2019.11.003

Fonseca CT, Oliveira SC, and Alves CC. 2015. Eliminating Schistosomes through Vaccination: What are the Best Immune Weapons? Front Immunol 6:95. 10.3389/fimmu.2015.00095

Giesemann T, Rathke-Hartlieb S, Rothkegel M, Bartsch JW, Buchmeier S, Jockusch BM, and Jockusch $\mathrm{H}$. 1999. A role for polyproline motifs in the spinal muscular atrophy protein SMN. Profilins bind to and colocalize with smn in nuclear gems. J Biol Chem 274:3790837914. 10.1074/jbc.274.53.37908

Hacariz O, Sayers G, and Baykal AT. 2012. A proteomic approach to investigate the distribution and abundance of surface and internal Fasciola hepatica proteins during the chronic stage of natural liver fluke infection in cattle. J Proteome Res 11:3592-3604. 10.1021/pr300015p

Halton DW. 2004. Microscopy and the helminth parasite. Micron 35:361-390. 10.1016/j.micron.2003.12.001

Hanna RE. 1980. Fasciola hepatica: glycocalyx replacement in the juvenile as a possible mechanism for protection against host immunity. Exp Parasitol 50:103-114. 10.1016/0014-4894(80)90012-0

Hanna RE, Anderson A, and Trudgett AG. 1988. Fasciola hepatica: studies on vaccination of rats and mice with a surface antigen prepared from fluke homogenate by means of a monoclonal antibody. Res Vet Sci 44:237-241.

Jang SI, Lillehoj HS, Lee SH, Lee KW, Lillehoj EP, Bertrand F, Dupuis L, and Deville S. 2011a. Montanide IMS 1313 N VG PR nanoparticle adjuvant enhances antigen-specific immune

Peer) reviewing PDF | (2020:06:49566:2:0:NEW 11 Nov 2020) 
395

396

397

398

399

400

401

402

403

404

405

406

407

408

409

410

411

412

413

414

415

416

417

418

419

420

421

422

423

424

425

426

427

428

429

430

431

432

433

434

435

436

437

438

439

440

441

442

443

444

445 responses to profilin following mucosal vaccination against Eimeria acervulina. Vet Parasitol 182:163-170. 10.1016/j.vetpar.2011.05.019

Jang SI, Lillehoj HS, Lee SH, Lee KW, Lillehoj EP, Bertrand F, Dupuis L, and Deville S. 2011b. Montanide ISA 71 VG adjuvant enhances antibody and cell-mediated immune responses to profilin subunit antigen vaccination and promotes protection against Eimeria acervulina and Eimeria tenella. Exp Parasitol 127:178-183. 10.1016/j.exppara.2010.07.021

Jang SI, Lillehoj HS, Lee SH, Lee KW, Lillehoj EP, Bertrand F, Dupuis L, and Deville S. 2011c. Mucosal immunity against Eimeria acervulina infection in broiler chickens following oral immunization with profilin in Montanide adjuvants. Exp Parasitol 129:36-41. 10.1016/j.exppara.2011.05.021

Kelley JM, Elliott TP, Beddoe T, Anderson G, Skuce P, and Spithill TW. 2016. Current Threat of Triclabendazole Resistance in Fasciola hepatica. Trends Parasitol 32:458-469. 10.1016/j.pt.2016.03.002

Korupolu RV, Achary MS, Aneesa F, Sathish K, Wasia R, Sairam M, Nagarajaram HA, and Singh SS. 2009. Profilin oligomerization and its effect on poly (L-proline) binding and phosphorylation. Int J Biol Macromol 45:265-273. 10.1016/j.jibiomac.2009.06.001

Krishnan K, and Moens PDJ. 2009. Structure and functions of profilins. Biophys Rev 1:71-81. 10.1007/s12551-009-0010-y

Kucera K, Koblansky AA, Saunders LP, Frederick KB, De La Cruz EM, Ghosh S, and Modis Y. 2010. Structure-based analysis of Toxoplasma gondii profilin: a parasite-specific motif is required for recognition by Toll-like receptor 11. J Mol Biol 403:616-629. 10.1016/j.jmb.2010.09.022

Kumpula EP, and Kursula I. 2015. Towards a molecular understanding of the apicomplexan actin motor: on a road to novel targets for malaria remedies? Acta Crystallogr F Struct Biol Commun 71:500-513. 10.1107/S2053230X1500391X

Kursula I, Kursula P, Ganter M, Panjikar S, Matuschewski K, and Schuler H. 2008a. Structural basis for parasite-specific functions of the divergent profilin of Plasmodium falciparum. Structure 16:1638-1648. 10.1016/j.str.2008.09.008

Kursula P, Kursula I, Massimi M, Song YH, Downer J, Stanley WA, Witke W, and Wilmanns M. $2008 \mathrm{~b}$. High-resolution structural analysis of mammalian profilin 2a complex formation with two physiological ligands: the formin homology 1 domain of mDia1 and the prolinerich domain of VASP. J Mol Biol 375:270-290. 10.1016/j.jmb.2007.10.050

LaCourse EJ, Perally S, Morphew RM, Moxon JV, Prescott M, Dowling DJ, O'Neill SM, Kipar A, Hetzel U, Hoey E, Zafra R, Buffoni L, Perez Arevalo J, and Brophy PM. 2012. The Sigma class glutathione transferase from the liver fluke Fasciola hepatica. PLoS Negl Trop Dis 6:e1666. 10.1371/journal.pntd.0001666

Lambrechts A, Jonckheere V, Dewitte D, Vandekerckhove J, and Ampe C. 2002. Mutational analysis of human profilin I reveals a second $\mathrm{PI}(4,5)-\mathrm{P} 2$ binding site neighbouring the poly(L-proline) binding site. BMC Biochem 3:12. 10.1186/1471-2091-3-12

Lammas DA, and Duffus WP. 1983. The shedding of the outer glycocalyx of juvenile Fasciola hepatica. Vet Parasitol 12:165-178. 10.1016/0304-4017(83)90005-5

LeJambre LF, Windon RG, and Smith WD. 2008. Vaccination against Haemonchus contortus: performance of native parasite gut membrane glycoproteins in Merino lambs grazing contaminated pasture. Vet Parasitol 153:302-312. 10.1016/j.vetpar.2008.01.032

Lu PJ, Shieh WR, Rhee SG, Yin HL, and Chen CS. 1996. Lipid products of phosphoinositide 3kinase bind human profilin with high affinity. Biochemistry 35:14027-14034. 10.1021/bi961878z

Mahoney NM, Janmey PA, and Almo SC. 1997. Structure of the profilin-poly-L-proline complex involved in morphogenesis and cytoskeletal regulation. Nat Struct Biol 4:953-960. 10.1038/nsb1197-953 
446

447

448

449

450

451

452

453

454

455

456

457

458

459

460

461

462

463

464

465

466

467

468

469

470

471

472

473

474

475

476

477

478

479

480

481

482

483

484

485

486

487

488

489

490

491

492

493

494

495

Mansilla FC, and Capozzo AV. 2017. Apicomplexan profilins in vaccine development applied to bovine neosporosis. Exp Parasitol 183:64-68. 10.1016/j.exppara.2017.10.009

Mares-Mejia I, Martinez-Caballero S, Garay-Canales C, Cano-Sanchez P, Torres-Larios A, Lara-Gonzalez S, Ortega E, and Rodriguez-Romero A. 2016. Structural insights into the IgE mediated responses induced by the allergens Hev b 8 and Zea $\mathrm{m} 12$ in their dimeric forms. Sci Rep 6:32552. 10.1038/srep32552

Mas-Coma S, Bargues MD, and Valero MA. 2018. Human fascioliasis infection sources, their diversity, incidence factors, analytical methods and prevention measures. Parasitology 145:1665-1699. 10.1017/S0031182018000914

Mas-Coma S, Valero MA, and Bargues MD. 2019. Fascioliasis. Adv Exp Med Biol 1154:71-103. 10.1007/978-3-030-18616-6_4

Merrifield M, Hotez PJ, Beaumier CM , Gillespie P, Strych U, Hayward T, and Bottazzi ME. 2016. Advancing a vaccine to prevent human schistosomiasis. Vaccine 34:2988-2991. 10.1016/j.vaccine.2016.03.079

Mittermann I, Fetrow JS, Schaak DL, Almo SC, Kraft D, Heberle-Bors E, and Valenta R. 1998. Oligomerization of profilins from birch, man and yeast: Profilin, a ligand for itself? . Sexual Plant Reproduction 11:183-191.

Moens PD, and Coumans JV. 2015. Profilin-1 mediated cell-cycle arrest: searching for drug targets. Cell Cycle 14:3669-3670. 10.1080/15384101.2015.1086204

Molehin AJ. 2020. Schistosomiasis vaccine development: update on human clinical trials. $J$ Biomed Sci 27:28. 10.1186/s12929-020-0621-y

Molina-Hernandez V, Mulcahy G, Perez J, Martinez-Moreno A, Donnelly S, O'Neill SM, Dalton JP, and Cwiklinski K. 2015. Fasciola hepatica vaccine: we may not be there yet but we're on the right road. Vet Parasitol 208:101-111. 10.1016/j.vetpar.2015.01.004

Moreau CA, Bhargav SP, Kumar H, Quadt KA, Piirainen H, Strauss L, Kehrer J, Streichfuss M, Spatz JP, Wade RC, Kursula I, and Frischknecht F. 2017. A unique profilin-actin interface is important for malaria parasite motility. PLoS Pathog 13:e1006412. 10.1371/journal.ppat.1006412

Moreau CA, Quadt KA, Piirainen H, Kumar H, Bhargav SP, Strauss L, Tolia NH, Wade RC, Spatz JP, Kursula I, and Frischknecht F. 2020. A function of profilin in force generation during malaria parasite motility that is independent of actin binding. J Cell Sci 134. 10.1242/jcs.233775

Munkhjargal T, Aboge GO, Ueno A, Aboulaila M, Yokoyama N, and Igarashi I. 2016. Identification and characterization of profilin antigen among Babesia species as a common vaccine candidate against babesiosis. Exp Parasitol 166:29-36. 10.1016/j.exppara.2016.03.024

Munn EA. 1997. Rational design of nematode vaccines: hidden antigens. Int J Parasitol 27:359366. 10.1016/s0020-7519(97)00003-9

Pinto-Costa R, and Sousa MM. 2020. Profilin as a dual regulator of actin and microtubule dynamics. Cytoskeleton (Hoboken) 77:76-83. 10.1002/cm.21586

Plattner F, Yarovinsky F, Romero S, Didry D, Carlier MF, Sher A, and Soldati-Favre D. 2008. Toxoplasma profilin is essential for host cell invasion and TLR11-dependent induction of an interleukin-12 response. Cell Host Microbe 3:77-87. 10.1016/j.chom.2008.01.001

Ravida A, Cwiklinski K, Aldridge AM, Clarke P, Thompson R, Gerlach JQ, Kilcoyne M, Hokke $\mathrm{CH}$, Dalton JP, and O'Neill SM. 2016. Fasciola hepatica Surface Tegument: Glycoproteins at the Interface of Parasite and Host. Mol Cell Proteomics 15:3139-3153. 10.1074/mcp.M116.059774

Skare P, Kreivi JP, Bergstrom A, and Karlsson R. 2003. Profilin I colocalizes with speckles and Cajal bodies: a possible role in pre-mRNA splicing. Exp Cell Res 286:12-21. $10.1016 / \mathrm{s} 0014-4827(03) 00102-2$

PeerJ reviewing PDF | (2020:06:49566:2:0:NEW 11 Nov 2020) 
496

497

498

499

500

501

502

503

504

505

506

507

508

509

510

511

512

513

514

515

516

517

518

519

520

521

522

523

524

525

526

527

528

529

530

531

532

533

534

535

536

537
Sobhon P, Anantavara S, Dangprasert T, Viyanant V, Krailas D, Upatham ES, Wanichanon C, and Kusamran T. 1998. Fasciola gigantica: studies of the tegument as a basis for the developments of immunodiagnosis and vaccine. Southeast Asian J Trop Med Public Health 29:387-400.

Stuven T, Hartmann E, and Gorlich D. 2003. Exportin 6: a novel nuclear export receptor that is specific for profilin.actin complexes. EMBO J 22:5928-5940. 10.1093/emboj/cdg565

Swan J, Sakthivel D, Cameron TC, Faou P, Downs R, Rajapaksha H, Piedrafita D, and Beddoe T. 2019. Proteomic identification of galectin-11 and -14 ligands from Fasciola hepatica. Int J Parasitol 49:921-932. 10.1016/j.ijpara.2019.06.007

Tanaka S, Kuroda Y, Ihara F, Nishimura M, Hiasa J, Kojima N, and Nishikawa Y. 2014. Vaccination with profilin encapsulated in oligomannose-coated liposomes induces significant protective immunity against Toxoplasma gondii. Vaccine 32:1781-1785. 10.1016/j.vaccine.2014.01.095

Tang X, Suo J, Li C, Du M, Wang C, Hu D, Duan C, Lyu Y, Liu X, and Suo X. 2018. Transgenic Eimeria tenella Expressing Profilin of Eimeria maxima Elicits Enhanced Protective Immunity and Alters Gut Microbiome of Chickens. Infect Immun 86. 10.1128/IAI.0088817

Toet H, Piedrafita DM, and Spithill TW. 2014. Liver fluke vaccines in ruminants: strategies, progress and future opportunities. Int J Parasitol 44:915-927. 10.1016/j.ijpara.2014.07.011

Wijffels GL, Sexton JL, Salvatore L, Pettitt JM, Humphris DC, Panaccio M, and Spithill TW. 1992. Primary sequence heterogeneity and tissue expression of glutathione Stransferases of Fasciola hepatica. Exp Parasitol 74:87-99. 10.1016/00144894(92)90142-w

Willadsen P, and Kemp DH. 1988. Vaccination with 'concealed' antigens for tick control. Parasitol Today 4:196-198. 10.1016/0169-4758(88)90084-1

Wilson RA, Wright JM, de Castro-Borges W, Parker-Manuel SJ, Dowle AA, Ashton PD, Young ND, Gasser RB, and Spithill TW. 2011. Exploring the Fasciola hepatica tegument proteome. Int J Parasitol 41:1347-1359. 10.1016/j.ijpara.2011.08.003

Wopfner N, Willeroidee M, Hebenstreit D, van Ree R, Aalbers M, Briza P, Thalhamer J, Ebner C, Richter K, and Ferreira F. 2002. Molecular and immunological characterization of profilin from mugwort pollen. Biol Chem 383:1779-1789. 10.1515/BC.2002.199

Yarovinsky F, Zhang D, Andersen JF, Bannenberg GL, Serhan CN, Hayden MS, Hieny S, Sutterwala FS, Flavell RA, Ghosh S, and Sher A. 2005. TLR11 activation of dendritic cells by a protozoan profilin-like protein. Science 308:1626-1629. 10.1126/science. 1109893

Zhang SM, Lv ZY, Zhou HJ, Zhang LY, Yang LL, Yu X, Zheng H, and Wu ZD. 2008. Characterization of a profilin-like protein from Schistosoma japonicum, a potential new vaccine candidate. Parasitol Res 102:1367-1374. 10.1007/s00436-008-0919-2 


\section{Figure 1}

Bioinformatic analysis of $F$. hepatica profilin (FhProfilin) gene.

A) The amino acid sequence for FhProfilin (accession number D915_008168;

GEVX010111281). The red letters indicate the profilin family domain. Black triangles indicate the putative actin-binding sites. $B, C$ ) Homology model of FhProfilin showed as a ribbon (B) and the crystal structure of Saccharomyces cerevisiae profilin (1YPR) (C). The residues involved in phosphatidylinositol phosphate interaction (light pink) and proline binding (light green). D) Phylogenetic tree of the $F$. hepatica profilin was produced online using Phylogeny.fr (Dereeper et al. 2008). The genebank or UniProtKB accession numbers used to construct the tree appear after each species name. Numbers shown at branch nodes indicate bootstrap values.

A

1 MSEEWCARCRSHVGDYKSLASLAFTGLDGNLYGNSDSTNFPVTVEFAKSAIAAFSGASPSSLTLNG 66

$67 \nabla_{\text {EKFIVLQKNDECLIGKCGRKTLFVYPCKSSCIFGLSVDTDESMNATNGNAACAMLSEEYKKLGLY }} \boldsymbol{\nabla}_{130}$

B

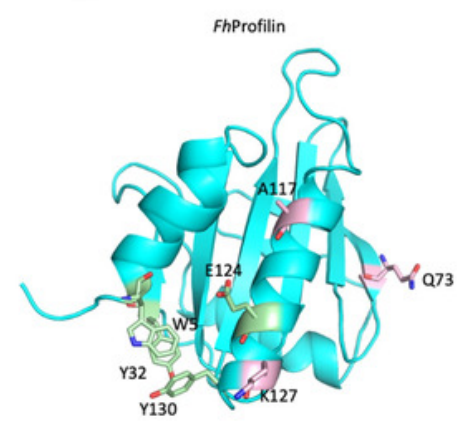

C

Yeast Profilin

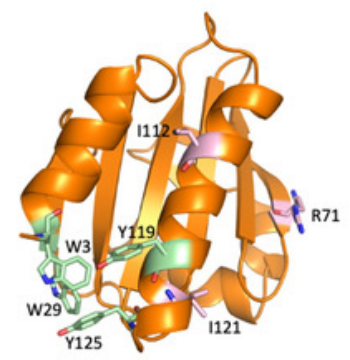

D

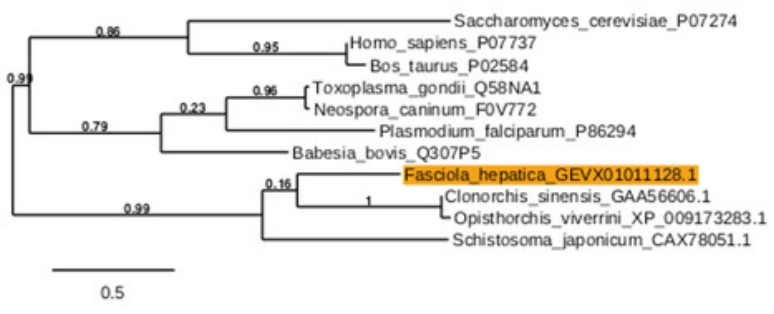


Figure 2

Recombinant expression and purification of FhProfilin

A) Cell lysate expressing FhProfilin were applied to a NI-IDA column and washed twice before elution with imidazole. $15 \mu \mathrm{l}$ of each stage of the purification was resolved by SDS-PAGE and stained with Coomassie blue. B) Size-exclusion chromatography trace of recombinant FhProfilin. Arrows indicate the elution volumes of proteins of known molecular weight. Insert: $15 \mu \mathrm{l}$ of each fraction was resolved by SDS-PAGE and stained with Coomassie blue. 
A

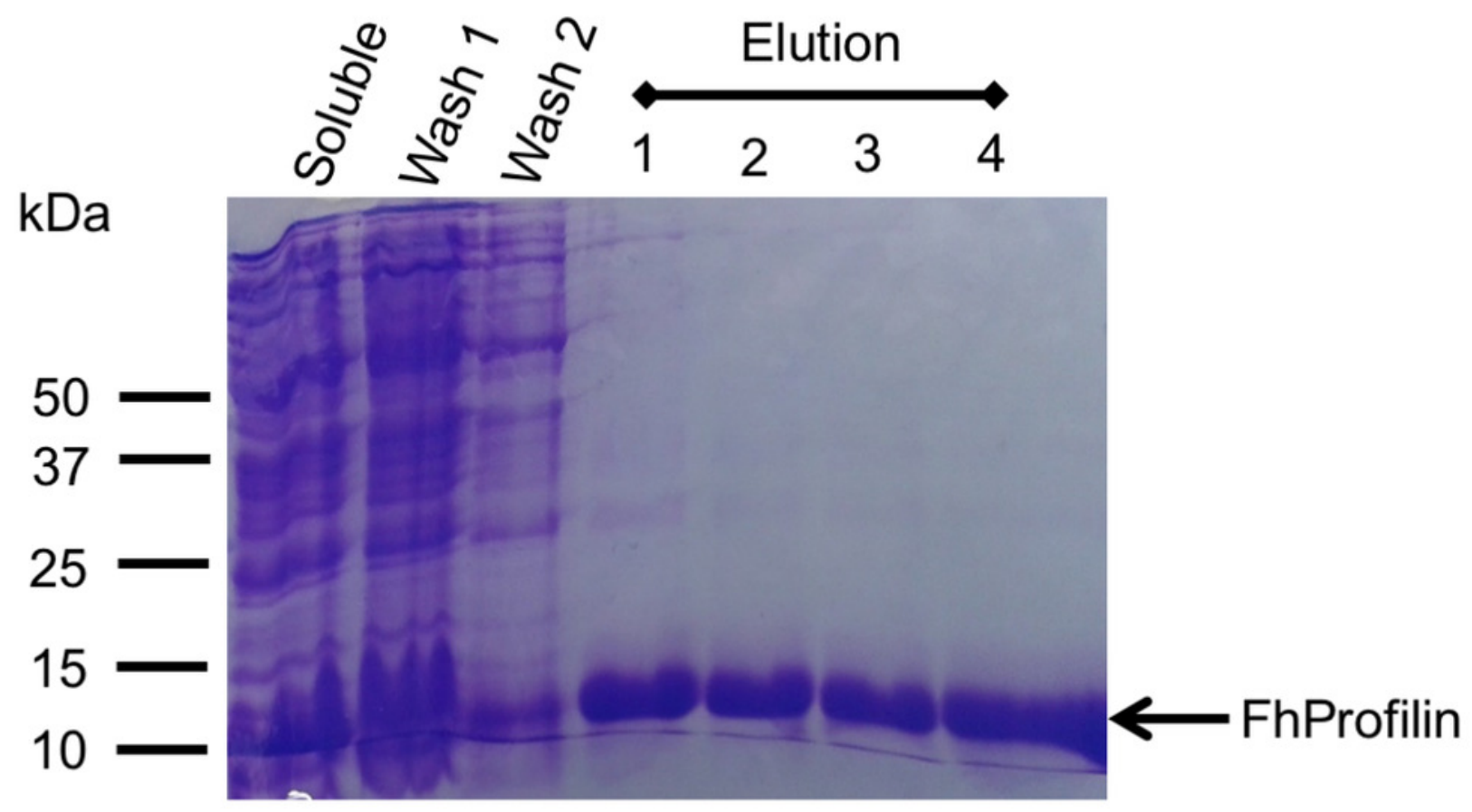

B

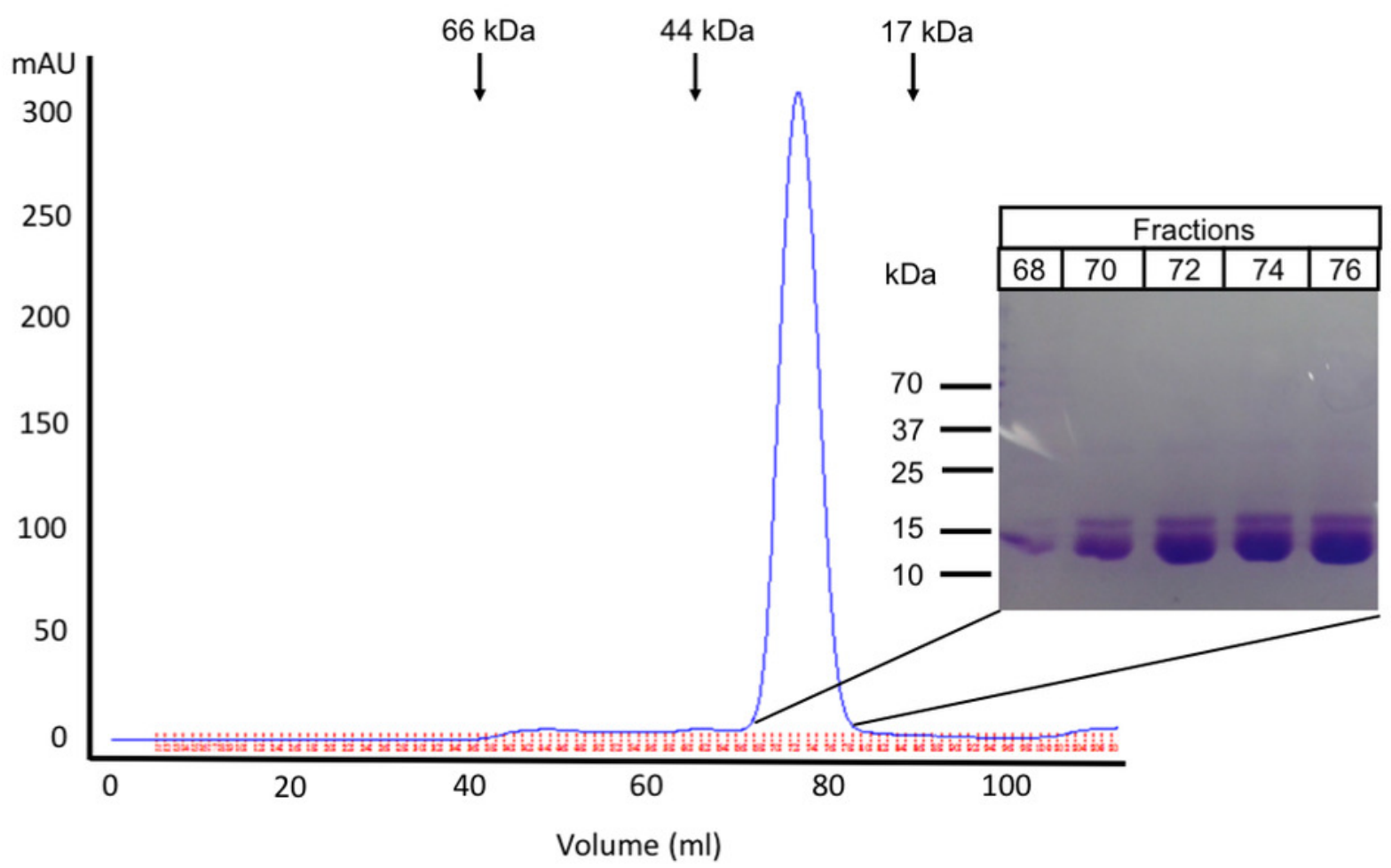


Figure 3

SDS-PAGE analysis of polymerized actin incubated with FhProfilin.

Different ratios of actin to FhProfilin (1:1, 1:2 and 1:4) were incubated and separated into polymerised or monomeric actin fractions by centrifugation. Control lanes contained no FhProfilin. The pellet $(\mathrm{P})$ and supernatant $(\mathrm{S})$ fractions $(15 \mu \mathrm{L})$ were resolved by SDS-PAGE and stained with Coomassie blue.

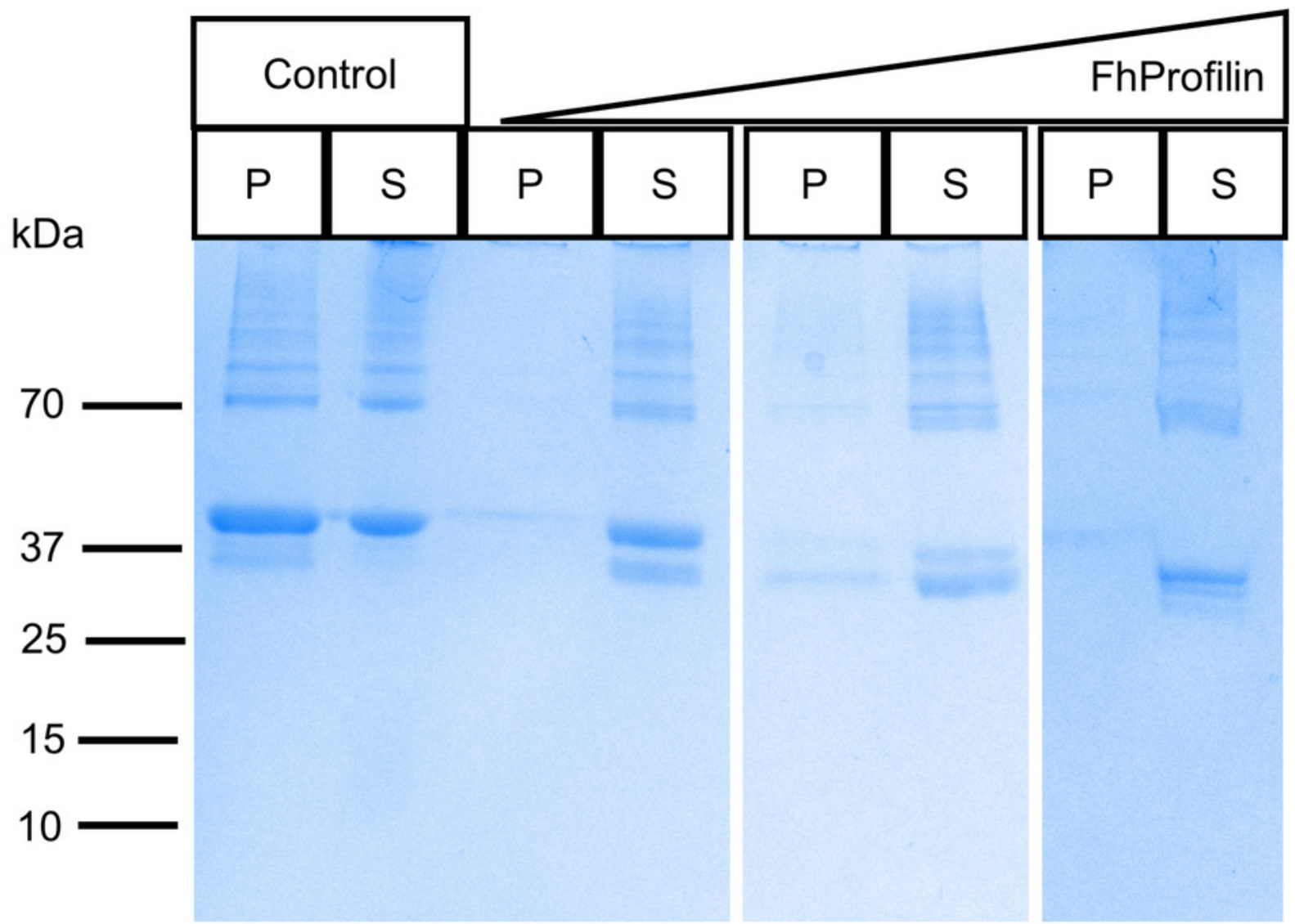


Figure 4

Binding of FhProfilin to phospholipids.

A) The location of the different phospholipids on the membrane (Echelon Biosciences Incorporated, USA). B) FhProfilin and C) GST-Grip respectively binding to phospholipid microarrays detected using an anti-hexhistidine and anti-GST antibody.

A

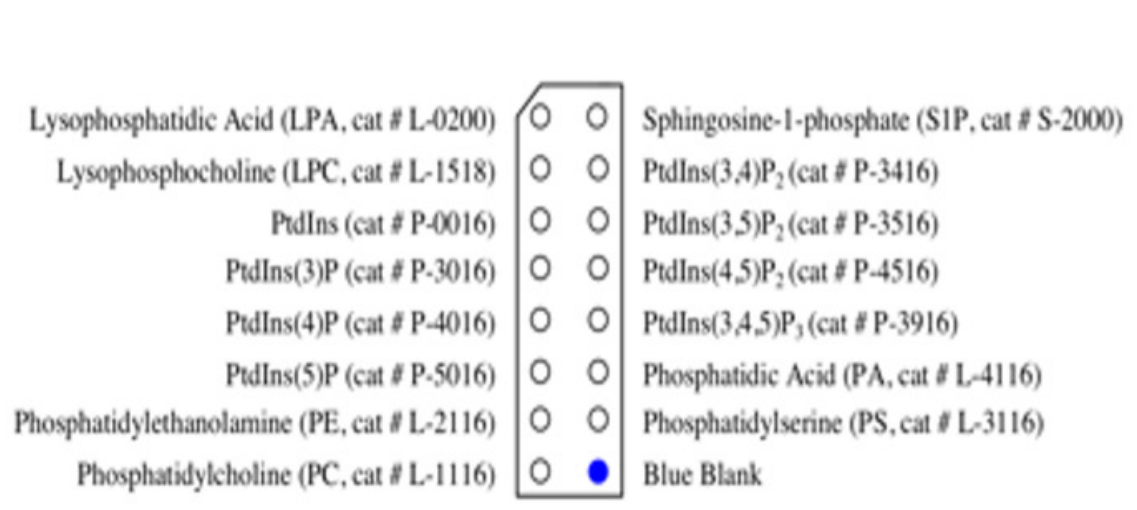

B

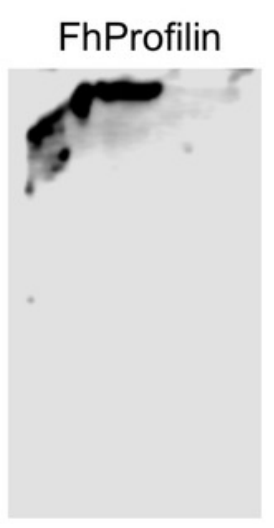

C

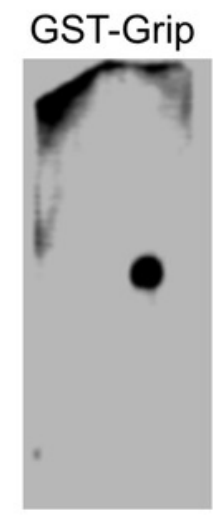




\section{Figure 5}

Binding of FhProfilin to polyproline sepharose.

Equal amounts of FhProfilin and BSA were passed over polyproline sepharose. Unbound (S) and Bound (B) proteins were visualized by SDS-PAGE and Coomassie staining. 


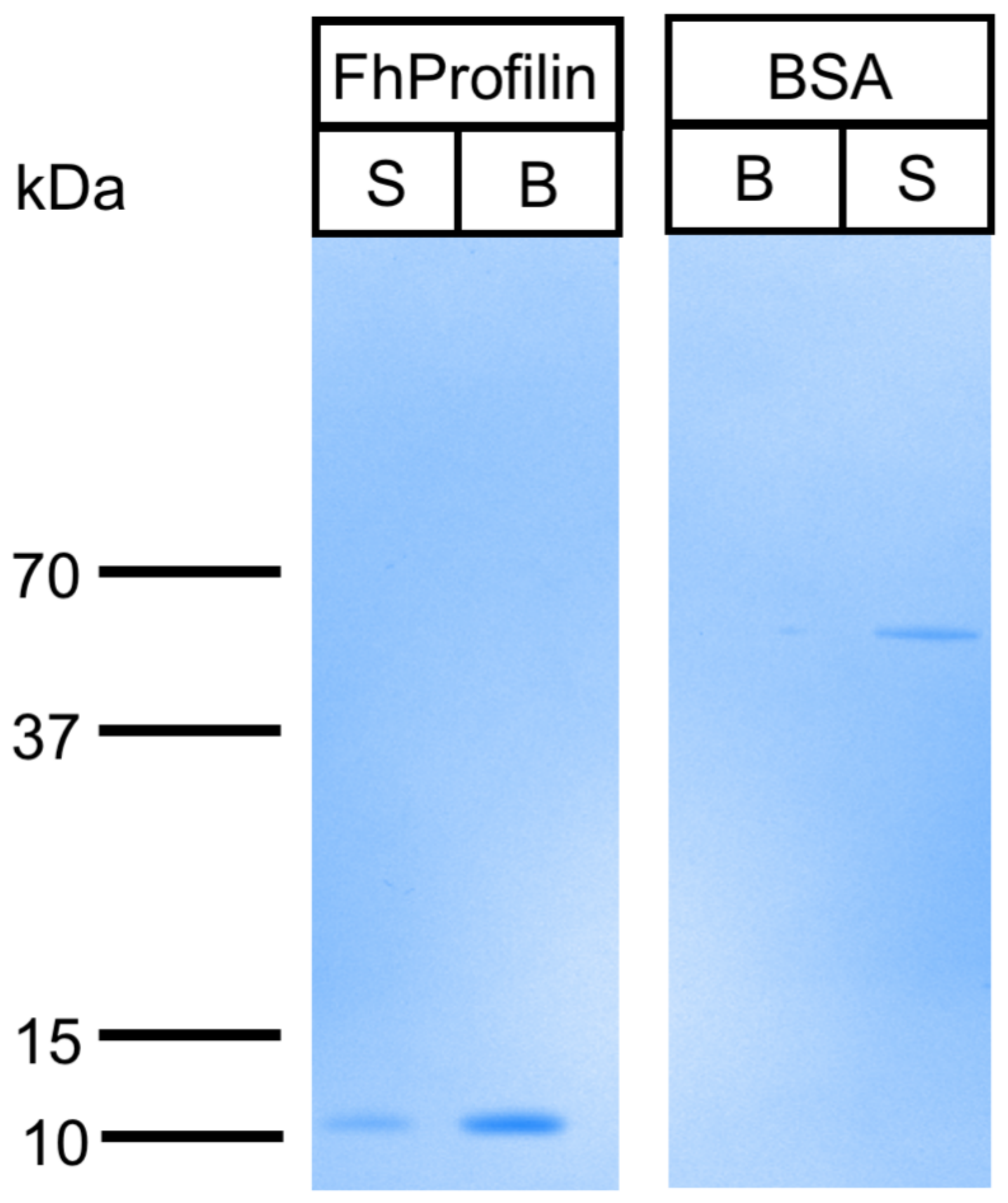


Figure 6

Western blot analysis of FhProfilin with immune sera.

Immune sera from infected ITT sheep (A), Merino sheep (B) and cattle (C) was probed on whole fluke extract (WE), recombinant FhProfilin (P) and native GST (G). The experiment was repeated a least 3 times.

A
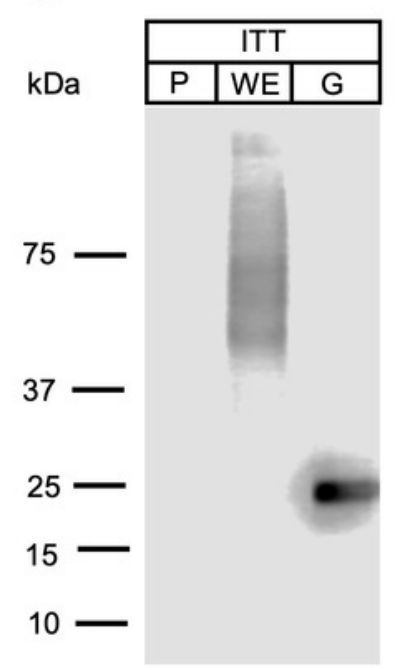

B

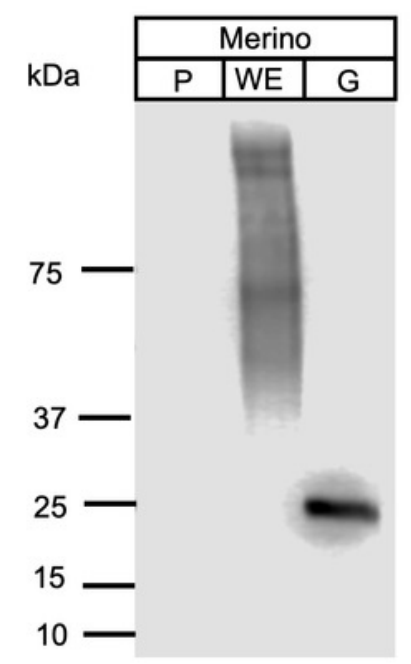

C

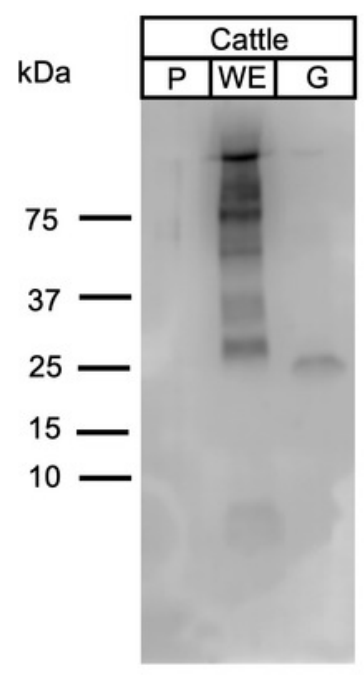

